\title{
Brave: what it means to be an Al Ethicist
}

\author{
Olivia Gambelin ${ }^{1}$
}

Received: 23 September 2020 / Accepted: 25 September 2020 / Published online: 6 October 2020

(c) Springer Nature Switzerland AG 2020

\begin{abstract}
Despite there being a strong call for responsible technology, the path towards putting ethics into action is still yet to be fully understood. To help guide the implementation of ethics, we have seen the rise of a new professional title; the AI Ethicist. However, it is still unclear what the role and skill set of this new profession must include. The purpose of this piece is to offer a preliminary definition of what it means to be an AI Ethicist by first examining the concept of an ethicist in the context of artificial intelligence, followed by exploring what responsibilities are added to the role in industry specifically, and ending on the fundamental characteristic that underlies it all: bravery.
\end{abstract}

Keywords AI Ethics · Ethical decision making · Bravery · Artificial intelligence

\section{Introduction}

You pause, hand on the door, to take a deep breath and run the talking points through your mind one last time before entering the room. On the other side of that door sits the board of directors, waiting patiently for your report on the ethical risks of a new AI system they are hoping to start developing, since it promises to turn a significant profit for the company. However, your ethical analysis of the proposed system has uncovered serious issues that would put the company at high risk for violating cultural values, while also destroying the reputation of the company in the process. You have prepared your report with meticulous detail, knowing full well that you will have to present an air-tight counter argument to the proposed AI system if you are to be heard. Even with the facts to back you up, you are aware that your points may jeopardize such an enormous profit and so could fall on deaf ears. Or worse, you may get through but trigger a negative reaction, resulting in your own dismissal. Being heard is only half the battle, because it does not matter if your argument gets through or not if no actions come out of it. What if the board hears you but still decides to go through with development? You are fully aware of this possibility, and that you will be blamed for when things go wrong even

Olivia Gambelin

olivia@ethicalintelligence.co

1 Ethical Intelligence Associates, Edinburgh, Scotland though you were the one that warned against it from the beginning.

With all of these thoughts and fears running through your mind, you stop to remind yourself of why you are here in the first place. You are an AI Ethicist and this situation, as stacked against you as the odds may seem, is the exact place that you are needed most. You were hired based on your skillset in ethics to be the voice in the room that says no when everyone else is silenced by a profit margin. But, as these kinds of moments prove to you time and time again, no amount of training, knowledge, or experience will matter if you are not brave enough to step into that room and open your mouth.

You take one last deep breath and push the door open, walking head on into what feels like an almost impossible situation. But you do it anyway, because you are brave.

\section{Framing the discussion}

In recent years, the field of AI Ethics has gained recognition as an essential component to building responsible technology. Alongside this growing movement, a new profession has emerged to help guide the implementation of ethics into technology: the AI Ethicist.

Although the role of an AI Ethicist may be listed as an essential position within the corporate structure [3], it still remains unclear as to what this role exactly entails and who is meant to fill it. Therefore, the purpose of this piece is to 
offer a preliminary definition of an AI Ethicist, providing the baseline from which we can move forward in refining what is to become a fundamental profession in the coming decades of technological development. To do such, I will start by stripping back the layers of context and simply define what it means to be an ethicist. From there, I will examine the concept of an ethicist in the context of artificial intelligence, followed by exploring what responsibilities are added to the role in industry specifically, and ending on the fundamental characteristic that underlies it all: bravery.

Before we can begin defining what it means to be an AI Ethicist, I must draw two key distinctions for clarity before engaging in the topic. First, it must be established that there is a clear difference between the field of AI Ethics and the profession of an AI Ethicist. The field of AI Ethics, generally speaking, encompasses a wide variety of profiles; from technologists and programmers, to evangelists and advocates, to policymakers and researchers, the common factor uniting this plethora of players being the intrinsic motivation to create responsible technology that pursues the human as the end beneficiary [3]. Think of AI Ethics as a puzzle, with each one of these profiles being a different piece, each one playing a vital role in contributing to the overall picture. This multidisciplinary perspective is crucial, as it enables a critical analysis of the ethical implications and consequences of artificial intelligence, and technology in general, to take place across discipline, culture, and mindset.

I draw this analogy to emphasize a clear distinction between the field and the profession, as the AI Ethicist is only one piece to the puzzle of AI Ethics. Because the AI Ethicist is named so closely after the field, there is a tendency to assume that only AI Ethicists are the ones at work in AI Ethics, that only they have the power to impact the change needed. This is not true, as can be seen by the brief list above of the other pertinent roles to the field. However, it is important to note that the AI Ethicist is an essential role, much like an edge puzzle piece to the overall picture, and so is worth defining in close detail. It is the specific role, and not the field, that I will, therefore, be focusing on in this piece.

The second distinction I must make is between the academic and the industry AI Ethicist. Beyond any doubt, the work and research being done in academia by those who would term themselves AI Ethicists is vitally important. However, my objective for this piece is to explore the title in the context of industry. An AI Ethicist is much easier to define within academia, as, generally speaking, they are the philosophers concerned directly with the ethical questions surrounding modern technology. The industry AI Ethicist, on the other hand, remains only a vaguely understood concept riddled with controversy. Therefore, it is in greater need of definition and will be the focus of this piece.

\section{Ethicist}

To understand the root of an AI Ethicist in industry, let us start by stripping away the layers to simply examine what it means to be an ethicist.

The term 'ethicist' is not a novelty label introduced only by the onset of the fourth revolution, ${ }^{1}$ instead it is a concept that has existed for centuries in a variety of contexts [5]. For example, we can turn to business or medical ethics, rich fields that have developed over the past decades to encompass guiding sets of principles, methodologies for applying these ethical codes, and experts who specialize in doing so. These experts are what we refer to as ethicists. Simply put, an ethicist is someone who applies abstract ethical principles and theories ${ }^{2}$ to concrete situations.

Based on the above definition, the first indicator of an ethicist is a command of ethical principles and theories. It is only by possessing this knowledge that the ethicist can move to apply these concepts to specific situations. Although this information is integral to the work of an ethicist, a comprehensive knowledge of the ethical principles relevant to the appropriate context does not determine a proficient ethicist. Sets of principles may at first appear to function as definitive facts, seeming to serve the purpose of providing clear objectives with hard set guides to action. If this were the case, then a strong command of the principles would be sufficient for the requirements of an ethicist. However, ethical principles instead are purposely abstract, meant not to denote precise normative actions but rather connote higher moral goods we strive to attain [5]. This high level of abstraction is essential to the applied nature of an ethicist's work, as it allows for the contextually sensitive analysis of principles per the demands of the distinct individual ethical dilemmas. Therefore, a knowledge of these principles and theories only partially satisfies the requirements of an ethicist.

Because ethical principles do not function as precise facts by which actions can be algorithmically deduced, the foundational indicator of an ethicist becomes the mastery of applying these abstract principles to concrete situations. Simply put, an ethicist must possess the ability to neutrally observe an ethically charged situation, abstract the details of such out to the higher ethical principles at play in order determine right from wrong, and then bring this decision

\footnotetext{
1 The fourth revolution, also known as the technological or digital revolution.

${ }^{2}$ For the purpose of this piece, 'ethical principles' refer to the higher moral principles that determine right from wrong as accepted by society. For example, the principles of transparency, accountability, and privacy are ethical principles in the larger context of artificial intelligence. On the other hand, 'ethical theories' refers to the different methods of applying these principles. For example, utilizing a consequentialist, deontologist, or virtue ethicist approach to the application.
} 
back down to contextually specific actions. This ability can also be referred to as the moral reasoning process [11]. Unlike common rational reasoning, which applies logic and theories to situations, moral reasoning is often heavily influenced by emotions and intuition. This can result in 'gut reactions' or decisions based not necessarily on facts and logic, but rather intuition and emotional response [9]. When taken by the layman, these moral decisions may appear to be irrational as they tend to prioritize different factors and decisions without explanation or awareness. The ethicist, on the other hand, is trained to recognize both the logical and illogical inputs during the moral reasoning process. This does not mean an ethicist is a morally superior individual who can separate emotion from ethically charged situations. On the contrary, it means that an ethicist must be cognitively aware of the different inputs, including the emotional, that are going into determining right from wrong. It is the ethicist's duty not to remove the emotional and intuitive input from the final decision, but instead to recognize its influence as a neutral third-party and utilize it as valid input alongside logic and facts for the final decision. This emotional intelligence combined with the mastery of applying abstract principles thus forms the skill set an ethicist must possess.

\section{Al Ethicist}

An ethicist is an individual with a robust knowledge of ethics who possesses the capacity to apply such abstract concepts to concrete situations. Returning the technical layer now, an AI Ethicist is someone who does this in the context of artificial intelligence. When laid out in this manner, the core of what it means to be an AI Ethicist becomes clear. In the context of artificial intelligence, and even technology at large, there exists an abundance of principles, codes and guidelines on both the corporate and policy levels that we strive to adhere our technology to [2]. Using these principles, the AI Ethicist is then able to move from abstract moral goods to determining right from wrong in the design, development and deployment of AI systems.

Of course, due to the rapidly growing nature of the field of artificial intelligence, the AI Ethicist must be proficient, undeniably, in the theory of the technology itself. An AI Ethicist cannot feasibly advise on artificial intelligence if he/she does not understand the capacities and limitations of the technology in the first place. To be clear, it is not the role nor to be expected of an AI Ethicist to be able to program the systems themselves. Instead, a strong understanding of aspects such as the difference between supervised and unsupervised learning, what it means to label a dataset, how consent of the user is obtained-essentially, how a system is designed, developed, and deployed-is necessary. In other words, an AI Ethicist must comprehend enough to be able to apprehend the instances in which key ethical questions must be answered.

It is also important to note that this addition of the context of artificial intelligence creates new issues that ethicists such as in business and medicine have not seen before. The extent to which the technology can both impact someone's life, as well as the sheer number of individuals it can affect, is staggering. The decisions one individual makes in the development of a single system, the imperceptible patterns of underlying societal norms in datasets, the unconscious bias of subgroups, are all amplified and intensified through AI systems [4]. Due to this scale of modern technology, ethicists are now expected to tackle ethical dilemmas that can cause significant changes in millions of lives halfway across the world from them. In other words, AI Ethicists are no longer dealing with person to person ethical issues, but rather machine to person. Therefore, it may be simple to conceptualize the addition of the context of AI to the foundational understanding of what makes an ethicist, however, this addition is highly significant as it is laden with a surplus of complex implications for the application of ethics and morality.

\section{Industry AI Ethicist}

Now that we have come to an understanding of what it means to be an ethicist in general, as well as specifically in the context of artificial intelligence, we can turn our attention to what it means to be an AI Ethicist in industry. In addition to the skillset of an ethicist and the understanding of the technology, an industry AI Ethicist must also exhibit an awareness of current policy developments, an understanding of business management and operations, and strong communication skills.

Ethics is not merely a matter of compliance, however knowledge of relevant policies and regulations in $\mathrm{AI}$ is still pertinent to the role of the industry AI Ethicist. As countries begin to implement legal requirements for artificial intelligence, companies become liable to these regulations. Currently, robust regulations for the development and usage of $\mathrm{AI}$ are in the early stages of formation, while the ethical requirements for AI exist only as recommended policies [2]. This means that the industry AI Ethicist must remain aware and updated on the legal developments surrounding AI, as these regulations provide the baseline standard that industry must meet. Of course, these policies and regulations should be regarded as only the bare minimum standard, as society's perception of ethical behavior often goes beyond what is legally required of a company [10]. In other words, a company can be legally compliant but still ethically questionable. An industry AI Ethicist thus must continually monitor developing relevant policies and regulations in $\mathrm{AI}$ to maintain an 
awareness of the baseline legal requirements on which he/ she can then build further ethical standards.

Once the necessary ethical standards have been established, the industry AI Ethicist must have a mind for business management and operations to then help guide the essential application of such standards. There are many methods for applying ethics in business, the majority of which involve the industry AI Ethicist working with the people and processes behind the technology instead of directly with the technology itself. For example, a popular method of application is ethical frameworks, internal structures designed for the people creating the technology to consistently check the ethical implications and consequences of their decisions in the product development lifecycle [6]. Without an understanding of how $\mathrm{AI}$ and data product lifecycles function from a project management perspective, the industry AI Ethicist risks complicating processes to the point of counter-productivity when trying to implement an ethical framework. This is but one example of a multitude of application methods, all of which illustrate the importance of an industry AI Ethicist understanding business management and operations to be able to effectively apply ethics to the technology being built.

Finally, in addition to an interdisciplinary knowledge of policy and business, the industry AI Ethicist must also possess the distinctive communication skills of active listening and empathic conveyance of sensitive topics. Strong communication skills are important for any profession, however, active listening and empathetic communication are especially important in the role of an industry AI Ethicist due to the tendency of defensive and emotional reactions towards ethically permeated situations [8]. Ethical issues are often complex, encompassing elements such as diverse sets of stakeholder opinions, cultural considerations, and emotionally charged situations, all of which require a certain degree of composure and ability to listen without projecting personal beliefs onto the circumstances. In other words, an industry AI Ethicist is expected to serve the role of a neutral body, someone who is brought in to actively listen and understand the facts of the issue to provide an answer as close to objective as possible. Additionally, the delivery of such answers is crucial, as it must be done in an empathic manner so as to avoid provoking defensive responses. Because ethical decision making is closely connected with perception of self, we are prone to defensiveness if one of our ethical decisions is being called into question [1]. By exhibiting empathy when conveying answers that are not necessarily comfortable to hear, the industry AI Ethicist is able to engage stakeholders on difficult topics surrounding ethical dilemmas. It does not matter how robust or intricate an ethical solution is if it cannot be communicated to the point of successful implementation, making the distinctive skills of active listening and empathetic communication essential to the role of the industry AI Ethicist.

\section{Bravery}

There are numerous attributes to an industry AI Ethicist, the core elements of which have been covered in this paper. To summarize these points into a comprehensive definition, an industry AI Ethicist is an individual with a robust knowledge of ethics who possesses the ability to apply and communicate ethical principles in the context of artificial intelligence within a corporate structure. Although this definition covers what is to be expected of an individual in this profession, there still remains one underlying characteristic that has become a defining factor of this role.

Intellectual capacities and emotional intelligence are essential, but above all else an AI Ethicist must be brave.

To be brave is to show courage in the face of fear. Returning back to the root definition of what makes an ethicist in the first place, we see the need for bravery in the face of moral ambiguity. Although an ethicist works with determined sets of ethical principles, the interpretations of these principles can drastically differ, which in turn affects how the principles are fulfilled. Simply put, there is no algorithmic approach to applying ethics, an uncertainty which often leads to fear of indistinct objectives in a world of data driven precision. The ethicist, however, does not shy away from this challenge and instead bravely embraces the opportunity to pursue the best possible solution that will uphold human values.

Again adding back the layer of artificial intelligence to the context, we see that one of the more significant problems in the technology industry has been the severe lack of accountability over decisions taken during development cycles that lead to wider consequences once the technology has been released. Because of the complexity of an AI system and the amount of people involved in its development, it becomes difficult to pinpoint the exact origin of responsibility. This phenomenon is known as the moral responsibility gap, which essentially occurs when there is no clear answer for who is to blame when a system does something wrong [7]. When an AI Ethicist comes into the equation, however, he/she becomes the default agent of responsibility. Much like a compliance officer in relation to regulations, it becomes expected of the AI Ethicist to uncover and fix ethically questionable situations and decisions before major consequences occur. If an ethical misstep does transpire, the ethicist is the first to be held accountable, just as the compliance officer is held accountable for a legal violation. However, unlike a compliance officer, there are no regulations in place requiring a company to follow the recommendations of the ethicist, which means the AI Ethicist faces the potential to be used as a scapegoat whether or not a company 
has followed their ethical recommendations. Thus, the AI Ethicist must be brave enough to shoulder responsibility for the ramifications of the technology, despite the lack of legal obligation a company has to follow through on any ethical recommendations.

Finally, looking at the context of industry, we see that more often than not, companies are driven by profit-related motivators, of which ethics is not necessarily one. Although the claim can be made that the ethical business is the one that will be more resilient in the long term, there are not yet clear metrics for how ethics can positively impact a profit margin. On the contrary, ethics is often placed within a blocker narrative, even to the extreme as being called a hindrance to innovation, as it becomes another set of limitations in addition to the technical ones that determine what is and is not possible for an AI system. What this means for the industry, AI Ethicist is that he/she must remain detached from the influences of shareholders and stakeholders alike, granting equal consideration to both, to be able to objectively examine decisions and systems. In other words, an AI Ethicist must be brave enough to walk into a room where he/ she knows he/she may very well be the only one saying no, stand by their decision, and have the patience to empathetically communicate with those in the room.

\section{Conclusion}

As is now evident, bravery is an essential characteristic of the AI Ethicist in this current day and age. Although these are but a few examples, bravery can be observed in many forms throughout all layers of both the definition and responsibilities of the industry AI Ethicist. In light of this, we must revise our preliminary definition of this developing profession:

What does it mean to an AI Ethicist in industry? It means to be an individual with a robust knowledge of ethics who possesses the ability to apply and communicate ethical principles in the context of artificial intelligence within the corporate structure, and to do it all with bravery.

\section{Compliance with ethical standards}

Conflicts of interest This is an opinion piece on the professional title "AI Ethicist" which is my personal title under such I engage in consulting activities as well as run the firm Ethical Intelligence Associates.

\section{References}

1. Albert, L.S., Reynolds, S.J., Turan, B.: Turning inward or focusing out? Navigating theories of interpersonal and ethical cognitions to understand ethical decision-making. J. Bus Ethics 130, 467-484 (2015). https://doi.org/10.1007/s10551-014-2236-2

2. Jobin, A., Ienca, M., Vayena, E.: The global landscape of AI ethics guidelines. Nat. Mach. Intell. 1, 389-399 (2019). https://doi. org/10.1038/s42256-019-0088-2

3. Shahriari, K., Shahriari, M.: IEEE standard review-Ethically aligned design: A vision for prioritizing human wellbeing with artificial intelligence and autonomous systems. IEEE Can. Int. Human. Technol. Conf. (IHTC) Toronto ON 2017, 197-201 (2017). https://doi.org/10.1109/IHTC.2017.8058187

4. Righetti, L., Madhavan, R., Chatila, R.: Unintended consequences of biased robotic and artificial intelligence systems [ethical, legal, and societal issues]. IEEE Robot. Autom. Mag. 26(3), 11-13 (2019). https://doi.org/10.1109/MRA.2019.2926996

5. Lantz, G. "Applied Ethics: What Kind of Ethics and What Kind of Ethicist?" J. Appl. Philos., vol. 17, no. 1, 2000, pp. 21-28. JSTOR, www.jstor.org/stable/24354107. Accessed 19 Sept. 2020.

6. Leikas, J., Koivisto, R., Gotcheva, N.: Ethical framework for designing autonomous intelligent systems. J. Open Innov. Technol. Mark. Complex. 5, 18 (2019)

7. Matthias, A.: The responsibility gap: Ascribing responsibility for the actions of learning automata. Ethics Inf. Technol. 6, 175-183 (2004). https://doi.org/10.1007/s10676-004-3422-1

8. Pelletier, K.L., Bligh, M.C.: The aftermath of organizational corruption: employee attributions and emotional reactions. J. Bus Ethics 80, 823-844 (2008). https://doi.org/10.1007/s1055 1-007-9471-8

9. Roeser, S.: Moral emotions and intuitions. Springer, Berlin (2010)

10. Seeger, M.W., HipfelS.J. "Legal versus ethical arguments." The debate over corporate social responsibility (2007): 155-166.

11. Zollo, L., Pellegrini, M.M., Ciappei, C.: What sparks ethical decision making? the interplay between moral intuition and moral reasoning: Lessons from the scholastic doctrine. J. Bus Ethics 145, 681-700 (2017). https://doi.org/10.1007/s10551-016-3221-8

Publisher's Note Springer Nature remains neutral with regard to jurisdictional claims in published maps and institutional affiliations. 